\title{
Polarized Electron Beams In The MEIC Collider Ring At JLab
}

\author{
F. Lin', Ya.S. Derbenev, V.S. Morozov, Y. Zhang \\ Thomas Jefferson National Accelerator Facility, Newport News, VA 23606, USA \\ E-mail: fangleiejlab.org, derbenevejlab.org, morozovejlab.org, \\ yzhangejlab.org,
}

\section{D.P. Barber}

Deutsches Elektronen-Synchrotron (DESY), 22607 Hamburg, Germany

E-mail: mpybar@mail.desy.de

\begin{abstract}
The nuclear physics program of the Medium-energy Electron-Ion Collider (MEIC) at the JLab requires a highly-polarized (over 70\%) electron beam with longitudinal polarization at the collision points. This can be achieved by arranging the equilibrium polarization direction to be vertical in the arcs of the figure- 8 shape ring and longitudinal at collision points. The rotation of the polarization is accomplished at each energy by using universal spin rotators, each of which consists of a set of solenoids and dipoles placed at the end of each arc. To reduce the spin-orbit depolarization effect due to the synchrotron radiation, spin matching to make the sections between the rotators in the long straights spin transparent has to be considered. We present the current universal spin rotator design, address various coupling compensation schemes for the solenoids, provide polarization configurations based on the spin rotators' layout, and briefly explore the use of continuous injection of electrons from the CEBAF into the MEIC collider ring for maintaining high equilibrium polarization. This continuous injection might be less demanding than spin matching.
\end{abstract}

XVth International Workshop on Polarized Sources, Targets, and Polarimetry

September 9-13, 2013

Charlottesville, Virginia, USA

1

Speaker 


\section{Introduction}

The MEIC electron ring at JLab is designed to preserve and manipulate a highly polarized electron beam as required by the nuclear physics programme: i) polarization of $70 \%$ or above, ii) longitudinal polarization at collision points, iii) polarization flipping at required frequencies [1]. To achieve these goals, various strategies from different points of view have been carefully considered and investigated as follows.

1. The Continuous Electron Beam Accelerator Facility (CEBAF) at JLab serves at a full energy (3-12 GeV) electron injector and polarization source $(>85 \%)$ of the MEIC electron ring. Two Wien filters installed in the CEBAF photo-injector can be set up carefully to ensure the proper polarization orientation at the injection point of the MEIC collider ring at all beam energies.

2. The polarization configuration is designed to be vertical in the arcs so as to minimize spin diffusion (i.e., depolarization) and longitudinal at the collision points, as shown in Figure 1. In all, four newly-developed $90^{\circ}$ universal spin rotators [2] are located at each end of the two arcs to perform this spin rotation in the whole energy range.

3. The desired spin flipping to reduce systematic uncertainties in the experiments can be implemented by changing the electron polarization at the source through the photoinjector driver laser. The bunch trains have alternate polarizations at required frequencies, as illustrated in Figure 2.

4. Rapid and high precision measurements of the electron beam polarization in the MEIC can be executed with Mott and Compton polarimeters. The existing Mott polarimeter at the CEBAF photo-injector can measure the source polarization (1-2\% precision) and assist in aligning the orientation of the electron polarization prior to the injection into the MEIC electron collider ring. For the MEIC electron collider ring, we will use a Compton polarimeter. This can make both rapid and non-destructive measurements. In addition, the Compton polarimeter can measure both transverse and longitudinal polarization.

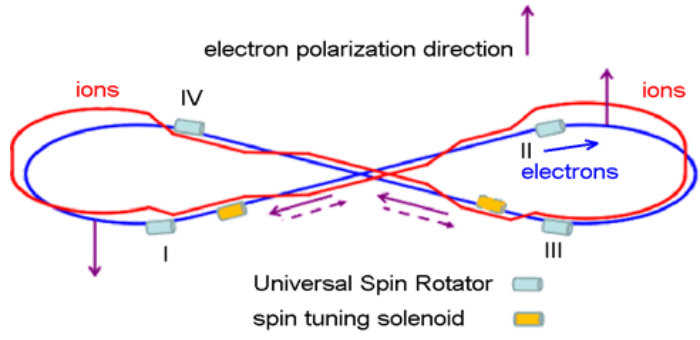

Figure 1: Polarization configuration in the MEIC electron collider ring.

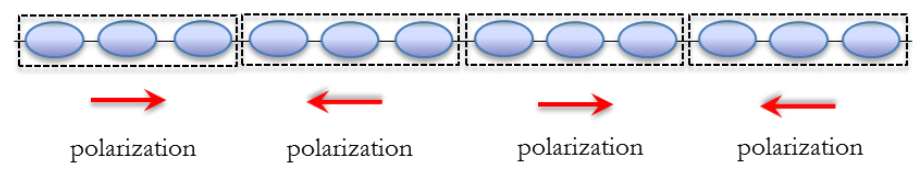

Figure 2: Alternate polarization of electron bunches from the CEBAF and in the MEIC collider ring. 
In principle, these strategies can guarantee that we meet the requirements for nuclear physics with the polarized electron beam. Our discussion in this paper concentrates on realistic considerations concerning the following related issues: spin rotator, depolarization due to spinorbit coupling and synchrotron radiation, polarization configuration and polarization lifetime.

\section{Spin Rotator}

As already mentioned, the spin rotators in the MEIC electron ring are the key means for rotating the polarization from the vertical in the arc to the longitudinal at the collision points and they should perform correctly over an energy range of 3 to $12 \mathrm{GeV}$. A universal spin rotator has been developed for this purpose [2], as shown schematically in Figure 3 [1]. This device includes two solenoids interleaved with two sets of arc dipole bends, which have $8.8^{\circ}$ and $4.4^{\circ}$ orbital bending angles, respectively. Accordingly, the spin rotation angles due to the two sets of dipole bends are the product of the corresponding bending angle and $G \gamma$, where $G=0.001159652$ is the gyromagnetic anomaly and $\gamma$ is the Lorentz factor. With proper solenoid magnetic fields, given in Table $1[1,2]$, the spin rotator produces a required $90^{\circ}$ spin rotation for all MEIC energies. Note that, such a spin rotator does not change the design orbit outside the rotators over the entire energy range.

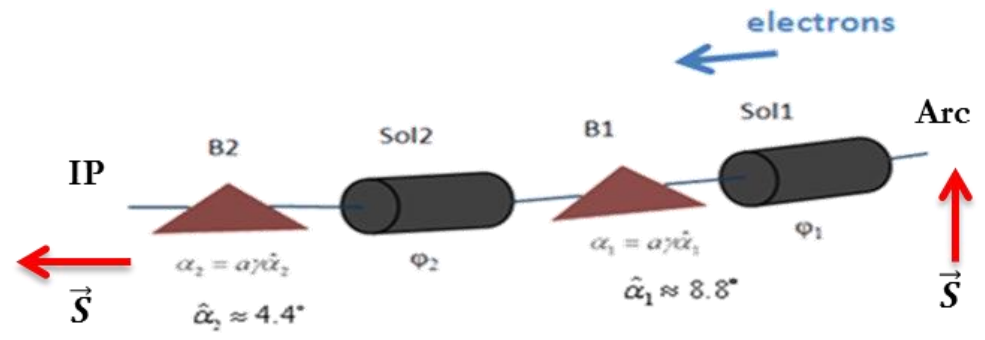

Figure 3: Schematic drawing of a universal spin rotator. B1 and B2 are the two sets of arc dipole bends rotating the spins around the vertical by $\alpha_{1}$ and $\alpha_{2}$. Sol1 and Sol2 are the solenoids rotating the spins around the longitudinal by $\varphi_{1}$ and $\varphi_{2}$.

\begin{tabular}{|c|c|c|c|c|c|c|}
\hline E & \multicolumn{2}{|c|}{ Solenoid 1 } & Arc Dipole 1 & \multicolumn{2}{|c|}{ Solenoid 2 } & Arc Dipole 2 \\
\hline & Spin Rotation & BDL & Spin Rotation & Spin Rotation & BDL & Spin Rotation \\
\hline $\mathrm{GeV}$ & $\mathrm{rad}$ & $\mathrm{T} . \mathrm{m}$ & $\mathrm{rad}$ & $\mathrm{rad}$ & T.m & $\mathrm{rad}$ \\
\hline 3 & $\pi / 2$ & 15.7 & $\pi / 3$ & 0 & 0 & $\pi / 6$ \\
\hline 4.5 & $\pi / 4$ & 11.8 & $\pi / 2$ & $\pi / 2$ & 23.6 & $\pi / 4$ \\
\hline 6 & 0.62 & 12.3 & $2 \pi / 3$ & 1.91 & 38.2 & $\pi / 3$ \\
\hline 9 & $\pi / 6$ & 15.7 & $\pi$ & $2 \pi / 3$ & 62.8 & $\pi / 2$ \\
\hline 12 & 0.62 & 24.6 & $4 \pi / 3$ & 1.91 & 76.4 & $2 \pi / 3$ \\
\hline
\end{tabular}

Table 1: Parameters of the spin rotator for MEIC at varius energies. 
However, the longitudinal solenoid field in the spin rotator can induce two problems: orbital coupling and polarization degradation due to spin-orbit coupling. The following two subsections will discuss these subjects and provide mechanisms to compensate and/or suppress these effects.

\subsection{Compensation of Transverse Orbital Coupling}

The longitudinal fields in the solenoids cause coupling between a particle's horizontal and vertical motion. Although such transverse coupling may be of benefit in the operation of synchrotrons to adjust the vertical emittance and increase the Touschek lifetime, the accompanying coupling resonances may reduce the available dynamic aperture for the particle motion and accordingly decrease the beam lifetime. Therefore, transverse orbital coupling is usually undesirable and should be compensated in a storage ring. Moreover, the solenoid fields in the spin rotators in the MEIC electron ring must be large in order to generate the required spin rotation.

Two techniques are commenly applied for compensating the orbital coupling introduced by solenoids. One was developed by Litvinenko and Zholents [3] using normal quadrupoles and an early optics for the MEIC was implemented in [4]. In this scheme, a solenoid is divided into two equal parts and an insert composed of normal quadrupoles is placed between the two parts of the solenoid. The quadrupole strengths are chosen so that for the whole combination (half solenoid + quadrupole insert + half solenoid) the $4 \times 4$ transport matrix is block-diagonal. Then the transverse coupling due to the solenoids is neutralized. The advantage of this scheme is that the quadrupole strengths are independent of the solenoid fields. The disadvantage is that each solenoid has to be divided into two identical parts, or two adjacent solenoids have to be identical. Otherwise, each individual solenoid needs one decoupling insert. This scheme may be proscribed due to costs and limitations on space.

A more straightforward alternative is to use skew quadrupoles distributed around the solenoid(s) region [5]. The coupling effect induced by the skew quadrupoles cancels that due to the solenoid(s) so that, again, the $4 \times 4$ transport matrix of the whole combination is blockdiagonal. That scheme places no requirements on the strengths and lengths of the various solenoids, while the skew quadrupole strengths depend on the solenoid strengths. This scheme might be more compact and less expensive than the aforementioned scheme using the normal quadrupoles.

Both schemes have been studied and implemented in the MEIC electron ring design. The spin rotator lattice functions with two different decoupling compensation techniques are plotted in Figure 4: the left plot is for the scheme with normal quadrupoles and the right one is for the scheme with skew quadrupoles. The horizontal dispersion (green) in both schemes has been suppressed in the solenoids to avoid the depolarization associated with transverse motion of offmomentum particles. Since the skew-quadrupole scheme can compensate the orbital coupling for two solenoids simultaneously, a spin rotator in this scheme (right in Figure 4) is about 10 meter shorter than that in the normal-quadrupole scheme (left in Figure 4). So, in total, 40 meter can be saved from four spin rotators in the MEIC electron collider ring. The choice will ultimately depend on the results of future polarization calculations. 

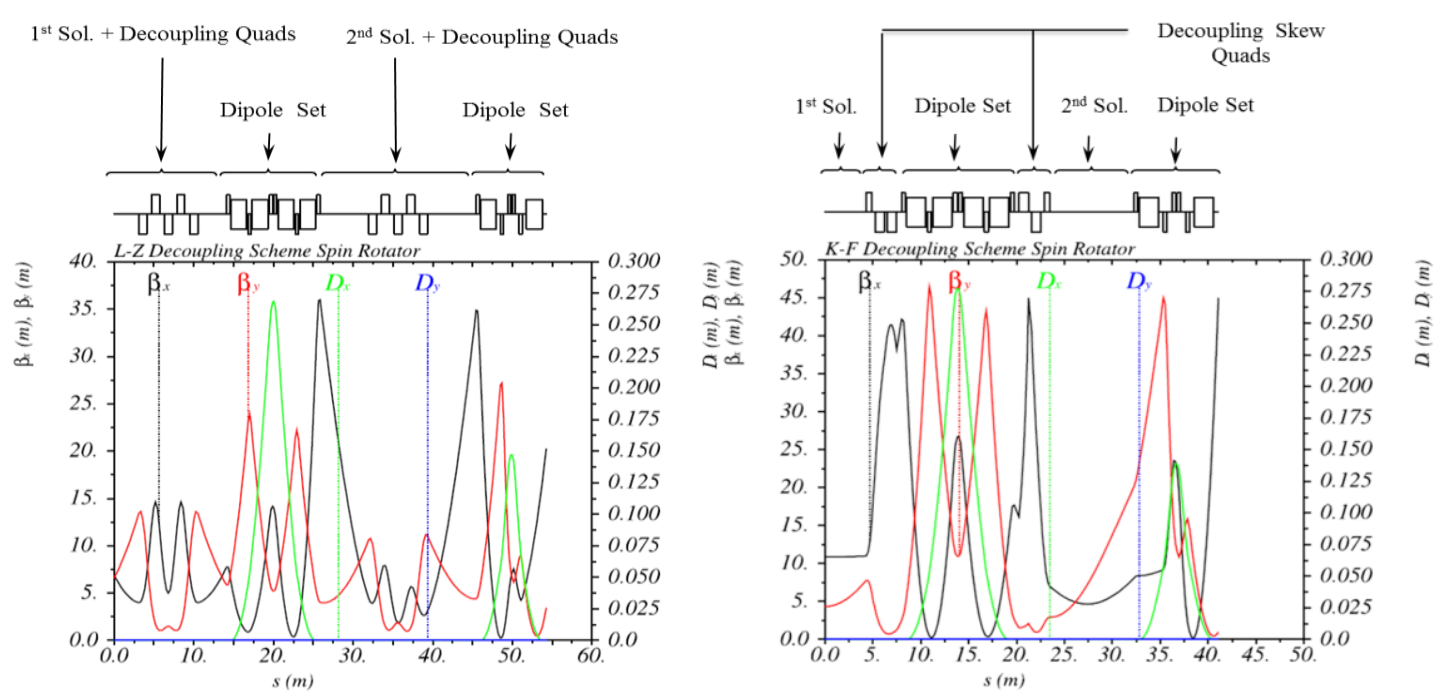

Figure 4: Spin rotator lattice functions with transverse coupling compensation using normal quadrupoles (left) and skew quadrupoles (right): horizontal beta function (black), vertical beta function (red), horizontal dispersion function (green), vertical dispersion function (blue).

\subsection{Polarization Degradation And Compensation}

A tiny fraction of the photons emitted as synchrotron radiation from relativistic electrons circulating in the vertical guiding field of an accelerator can cause spin flip from up to down and vice versa. However, the different flipping rates, the up-to-down and down-to-up, cause the electron beam to become spin polarized anti-parallel to the guiding magnetic field. This so called "Sokolov-Ternov" (S-T) self-polarization process [6] can provide a maximum polarization of $P_{s t}=92.4 \%$ in ideal circumstances on a time scale of a few minutes to a few hours. This is a much longer time scale than for other dynamical phenomena occurring in an accelerator. In addition to the flipping, the spin precesses in the external electric and magnetic fields along a trajectory according to the Thomas-BMT equation [7,8].

The majority of the emitted photons add a stochastic component to the electron orbital motion and determine the phase space density distribution. Moreover, the stochastic motion together with the spin-orbit coupling embodied in the Thomas-BMT equation can result in spin diffusion, and thus depolarization. This can be strongly enhanced in sections where the polarization is horizontal, by misalignments of the elements in an accelerator and/or special magnet systems like the spin rotators. The rate of depolarization rises with the beam energy and it increases faster than the S-T self-polarization effect. Therefore, the equilibrium polarization is usually less than $P_{s t}(92.4 \%)$ and depends on the relative strengths of the self-polarization and depolarization processes. Analytical estimates of the equilibrium polarization are given by the Derbenev-Kondratenko (D-K) formalism [9].

As mentioned above, the MEIC conceptual design adopted the solenoid spin rotators for providing the longitudinal polarization at the IPs and the vertical polarization in the arcs. These rotators have longitudinal body fields and radial end fields in the solenoids, as well as the fields in the dipoles and quadrupoles. The total rotation angle in one spin rotator is $90^{\circ}$ only for onmomentum particles with $\delta=\mathrm{dp} / \mathrm{p}=0$. For $\delta \neq 0$ particles, a spin perturbation to the off-momentum particles appears if the net integral of the solenoid fields is non-zero. Therefore, the geometry 
and optics of the ring have to be constructed in a proper way to eliminate such a pernicious influence on the polarization. So the well-known "spin matching" technique $[10,11]$ has been considered and used to suppress spin diffusion from some key regions by tuning and/or arranging the optics elements around the ring. These matters are discussed in the next section.

\section{Polarization Configuration}

The MEIC electron ring has a total of four spin rotators. They are located at each end of the two arcs, i.e., at the ends of the two long straights. See Figure 5. Each spin rotator has two solenoids, which have different lengths and strengths but the same field directions. All spin rotators have a similar structure, except that the solenoid fields at the beginning and the end of the long straights may have opposite signs. As a result the polarization configuration is determined by the solenoid field directions in the pair of spin rotators in the same long straight, and can be classified according to the two situations as follows.

Figure 5 depicts the figure- 8 shape MEIC electron ring and the polarization orientation in one long straight section (the green box). Pairs of spin rotators in the same long straight have the same solenoid polarities (blue arrows in the cylinders). To demonstrate the polarization orientation, two opposite longitudinal polarizations (black solid and dashed arrows) at the IPs have been depicted, corresponding to when the polarization is flipped at the source. In this configuration, the S-T self-polarization effect may help preserve one electron polarization state only, shown as the black solid arrow, because the polarization is anti-parallel to the vertical guiding fields in the two arcs. For the other electron polarization state shown as the black dashed arrow, the polarization is reversed at the IP and parallel to the guiding fields in the two arcs. Then the S-T effect will drive the polarization back, through zero, to the above polarization state of the black solid arrow. Therefore, if the polarization is flipped at the source, the polarization (black solid arrow) can be stable in some beam bunch trains if other sources of depolarization can be minimized, while the polarization (black dashed arrow) is not static in the other bunch trains.

In this type of polarization configuration, the same longitudinal solenoid field directions in the pair of spin rotators in the same long straight results in a non-zero net integral for the solenoid fields. This unavoidably induces a spin perturbation to the off-momentum particles. Equilibrium polarization and lifetime have been calculated using the code SLICK $[10,11]$ and this shows that the resulting rate of depolarization is larger than the rate for the S-T selfpolarization. Therefore, the beam is completely depolarized in a short time and the polarization lifetime, with highly polarized injected beams, is too short for a detector to collect enough data. The antidote to such a spin perturbation is spin matching, i.e. tuning the magnet elements, such as dipoles and quadrupoles, to get spin transparency at $1^{\text {st }}$ order in some key regions. Depolarization due to synchrotron motion tends to be particulary strong and dominant since the small synchrotron tune implies small phase advances. Then spin matching to overcome this source of spin perturbations in the straights and rotators is usually difficult and needs many tuning knobs to achieve this goal. This will burden the ring optics design.

Another polarization configuration is demonstrated in Figure 6, with a similar schematic drawing of the figure- 8 shape MEIC electron ring and polarization orientation in one long 
straight section (the green box). Pairs of spin rotators in the same long straight have the opposite solenoid polarities (blue arrows in the cylinders). As in Figure 5, in the demonstration of polarization orientation, two opposite longitudinal polarizations (black solid and dashed arrows) at the IPs also have been considered. In this type of polarization configuration, the polarization is anti-parallel to the vertical guiding field in one arc and parallel to the guiding field in the other one, regardless of the polarization direction at IPs. Hence the S-T process has a net depolarization effect in the whole collider ring, and all beam bunch trains from the polarized source will be similarly confronted and have a similar size of polarization in both polarization states.

In addition, with opposite longitudinal solenoid field directions of pairs of spin rotators in the same long straight, the net field integral is zero. Accordingly, at $1^{\text {st }}$ order the spin perturbation in the solenoids for off-momentum particles vanishes, thereby significantly reducing the burden on the spin matching and ring-optics design. Though the equilibrium polarization is zero in this polarization configuration, with highly polarized injected beams, the polarization lifetime at low energies is much longer (comparable to the beam lifetime) than in the first polarization configuration without any further effort on spin matching. This may be a challenge at higher energies because the polarization lifetime is mainly determined by the S-T depolarization effect and its lifetime is inversely proportional to the $5^{\text {th }}$ power of the Lorentz factor. The solution will be discussed in the next section on Continuous Injection.

The characteristics of the two polarization configurations in the MEIC electron collider ring have been itemized in Table 2. Both polarization configurations have their advantages and disadvantages. At lower energies $(<9 \mathrm{GeV})$, the polarization configuration II is a proper choice, with its reasonably large polarization lifetime for both polarization states and much less arduous spin matching. At higher energies (> $9 \mathrm{GeV}$ ), the small S-T depolarization time leaves no polarization for either polarization state. The polarization configuration I may maintain one polarization state at all energies as long as spin matching is performed suscessfully. However, this is usually a tedious task and may not be practical over the whole energy range.

\begin{tabular}{|c|c|}
\hline $\begin{array}{c}\text { Polarization Configuration I } \\
\text { same solenoid field directions in the same long straight }\end{array}$ & $\begin{array}{l}\text { Polarization Configuration II } \\
\text { Opposite solenoid field directions in the same long straight }\end{array}$ \\
\hline $\begin{array}{l}\text { - The Sokolov-Ternov effect may help to } \\
\text { preserve one polarization state with spin } \\
\text { matching. } \\
\text { - Spin matching is needed to maintain the } \\
\text { polarization due to the non-zero integral of } \\
\text { longitudinal solenoid fields in the two spin } \\
\text { rotators in the same long straight. } \\
\text { - The total depolarization time is determined } \\
\text { by the spin-orbit coupling depolarization } \\
\text { time. } \\
\left.\text { The design-orbit spin tune ( } v_{0}\right) \text { is not zero, } \\
\text { only because of the non-zero integral of the } \\
\text { longitudinal fields. }\end{array}$ & $\begin{array}{l}\text { - The Sokolov-Ternov effect does not contribute } \\
\text { to preserving the polarization. } \\
\text { - Spin matching is much less demanding due to } \\
\text { the zero integral of the longitudinal solenoid } \\
\text { fields in the two spin rotators in the same long } \\
\text { straight. } \\
\text { - The total polarization time is determined by the } \\
\text { Sokolov-Ternov depolarization time. } \\
\text { - The design-orbit spin tune }\left(v_{0}\right) \text { is zero, but can } \\
\text { be adjusted easily using weak fields because of } \\
\text { the figure- } 8 \text { shape. }\end{array}$ \\
\hline
\end{tabular}

Table 2: Characteristics of two polarization configurations. 


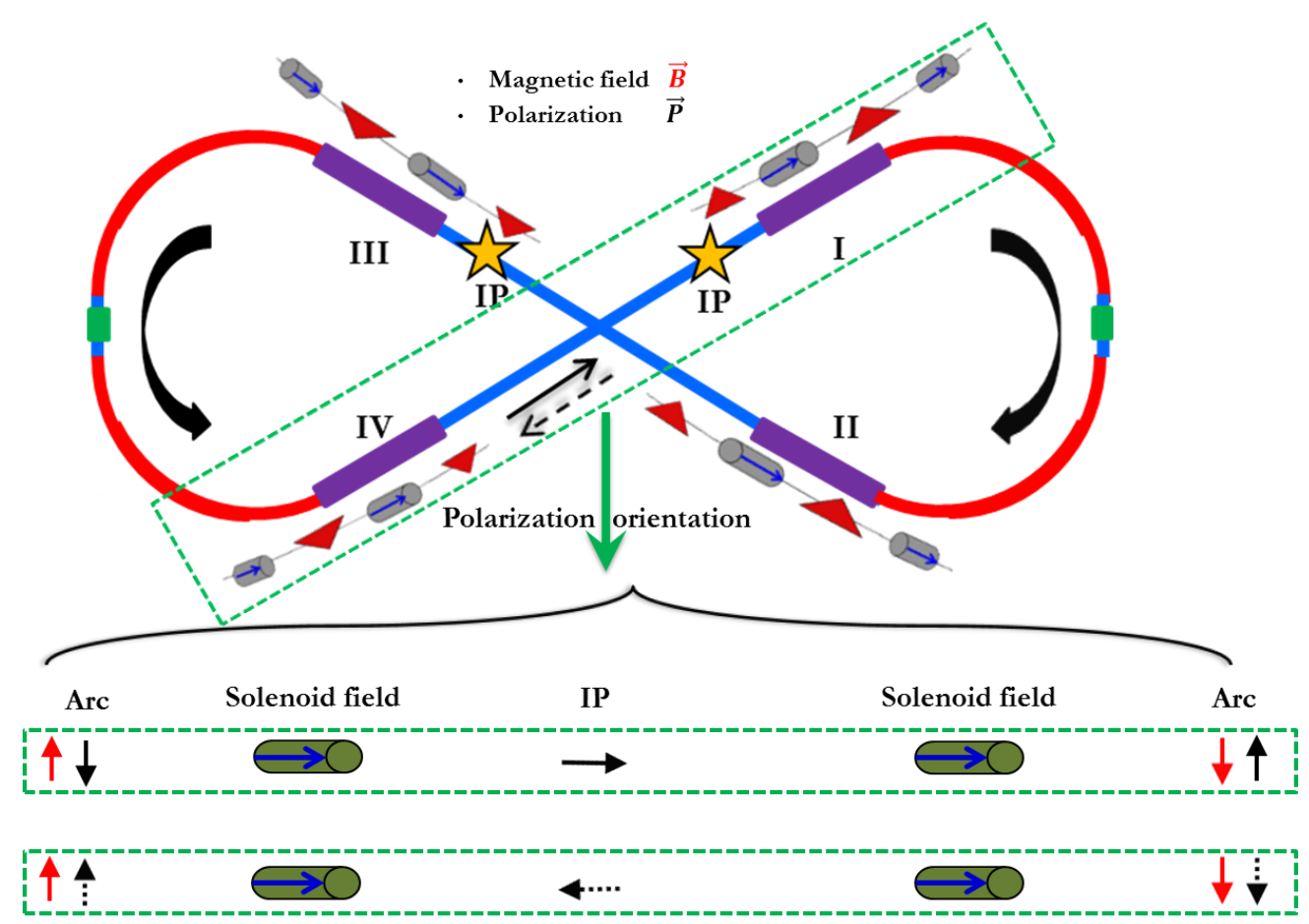

Figure 5: Polarization ( $\vec{P}$, black arrow) directions flip over in two arcs for the same longitudinal solenoid field directions in the same long straight. The blue arrow in the solenoid represents the field direction. The polarization orientation in one of the two long straights (shown in the green box) is demonstrated under the half brace with two different polarization states at the IP.

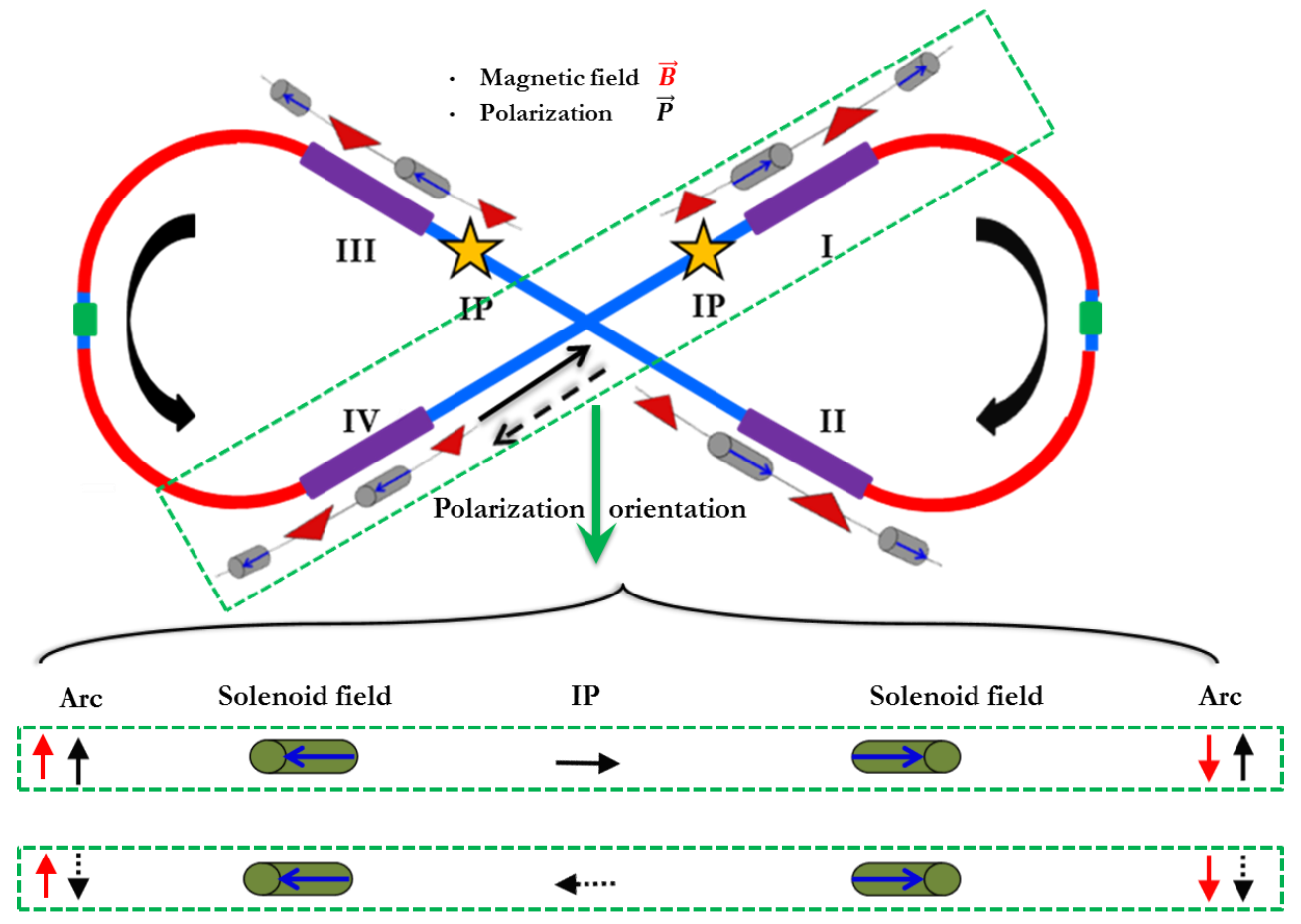

Figure 6: Polarization ( $\vec{P}$, black arrow) directions remain unchanged in the two arcs by having opposite longitudinal solenoid field directions in the same long straight. The blue arrow in the solenoid represents the field direction. The polarization orientation in one of the two long straights (shown in the green box) is demonstrated under the half brace with two different polarization states at the IP. 


\section{Continuous Injection}

An interesting solution for making the polarization configuration II work over the whole energy range in the MEIC electron ring is continuous injection of polarized electron beams from the CEBAF. Continuous injection, also named Top-off injection, has already been successfully applied in PEP-II at SLAC [12] to increase the integrated luminosity and will be used in the NSLS-II at BNL [13] to maintain the beam current. With the current state of the art, it is not a technical problem. The function of continuous injection in the MEIC is three-fold. First, the equlibrium polarization is maintained by mixing the old partly depolarized circulating beam with fresh highly polarized beam through continuous injection. Then at a constant stored beam current

$$
\frac{P_{e q u}}{P_{0}}=\frac{\frac{I_{i n j}}{I_{r i n g}} f_{r e v}}{\frac{1}{\tau_{d k}}+\frac{I_{i n j}}{I_{r i n g}} f_{r e v}},
$$

where $P_{e q u}$ is the attained equilibrium polarization, $P_{o}$ is the injected polarization, $I_{i n j}$ is the average injected beam current, $I_{\text {ring }}$ is the stored beam current, $f_{\text {rev }}$ is the revolution frequency, and $\tau_{d k}$ is the $\mathrm{D}-\mathrm{K}$ polarization lifetime. Though the $\mathrm{D}-\mathrm{K}$ polarization lifetime $\tau_{d k}$ in the current MEIC design for polarization configuration II is 1000 s and 240s at 9 and $12 \mathrm{GeV}$, respectively, calculated using the code SLICK, Eq. (1) shows that a 100nA average injected beam current from the CEBAF can easily maintain the equilibrium polarization $P_{\text {equ }}$ above $78 \%$ (assuming $P_{o}=80 \%$ ) in the collider ring. If necessary, some extraction may be introduced to avoid beam blow up. Second, with continuous injection, any particle losses due to the Touschek and/or IBS effect can be easily compensated and a constant beam current can be achieved. Third, a large momentum acceptance may not be required because particles do not need to circulate in the collider ring for a very long time. A decent momentum acceptance can help particles' extraction. This will significantly reduce the burden of handling the nonlinear beam dynamics.

Technical aspects and beam dynamics studies of the continuous injection will be discussed in the future work.

\section{Conclusion}

In conclusion, the MEIC electron collider ring at Jefferson Lab is designed to provide highly polarized electron beams for the nuclear physics program. Four solenoid spin rotators perform polarization rotation from the vertical in the arcs to the longitudinal at the IPs to satisfy the requirements for polarization preservation and requirements for the detectors. Two polarization configurations have been classified according to the arrangement of the spin rotators in the same long straights. With a continuous injection from the CEBAF, a very high polarization (>78\%) can be easily maintained in the MEIC collider ring, which requires the continuously injected current at 100nA level only. 


\section{Acknowledgements}

This work was authored by Jefferson Science Associates, LLC under U.S. DOE Contract No. DE-AC05-06OR23177 and DE-AC02-06CH11357. The U.S. Government retains a nonexclusive, paid-up, irrevocable, world-wide license to publish or reproduce this manuscript for U.S. Government purposes. We are grateful to A.M. Kondratenko, Y.N. Filatov and M.A. Kondratenko for their advice and discussions.

\section{References}

[1] S. Abeyratne et al., Science requirements and conceptual design for a polarized medium energy electron-ion collider at Jefferson lab, edited by Y. Zhang and J. Bisognano, arXiv:1209.0757 [physics.acc-ph] (2012).

[2] P. Chevtsov et al., Electron-ion collider spin manipulation system and its mathematics, JLabTN-10-026 (2010).

[3] A. Zholents et al., On the compensation of solenoid field effects by quadrupole lenses, BINP (Novosibirsk) Preprint 81-80 (1981). English translation: DESY Report L-Trans 289 (1984).

[4] H. Sayed et al., Spin rotator optics for MEIC, in proceedings of 2010 International Particle Accelerator Conference (IPAC'10), Kyoto, Japan (2010), TUPEB044, p.1626.

[5] Y. N. Filatov et al., Polarized proton beam acceleration at nuclotron with the use of the solenoid Siberian snake, in proceedings of $20^{\text {th }}$ International Spin Physics Symposium (SPIN 2012), Dubna, Russia, September 2012.

[6] A. A. Sokolov et al., On polarization and spin effects in synchrotron radiation theory, Sov. Phys. Dokl. 8: 1203 (1964).

[7] L. H. Thomas, The kinematics of an electron with an axis, Philos. Mag. 3, 1-22 (1927).

[8] V. Bargmann et al., Precession of the polarization of particles moving in a homogeneous electromagnetic field, Phys. Rev. Lett. 2, 435-436 (1959).

[9] Ya. S. Derbenev et al., Polarization kinematics of particles in storage rings, Sov. Phys. JETP. 37, 968 (1973).

[10] D. P. Barber et al., Handbook of Accelerator Physics and Engineering, edited by A. W. Chao and M. Tigner, World Sci., ${ }^{\text {st }}$ edition, $3^{\text {rd }}$ printing (2006).

[11]F. Lin et al., Electron polarization in the medium-energy electron-ion collider at JLAB, in the proceeding of 2012 International Particle Accelerator Conference (IPAC'12), New Orleans, Louisiana, USA (2012), TUPPC098, p.1386.

[12] J. L. Turner et al., Trickle-charge: a new operational mode for PEP-II, in the proceeding of $9^{\text {th }}$ European Particle Accelerator Conference (EPAC'04), Lucerne, Switzerland (2004), p. 881.

[13] T. Shaftan et al., NSLS-II injection concept, in the proceeding of 2005 Particle Accelerator Conference(PAC'05), Knoxville, Tennessee, USA (2005), p. 3408. 